\author{
Raimund Kastler \\ mit Beiträgen von \\ Matthias Pfisterer
}

\title{
Oberflächenfunde des Jahres 2005 im Bereich von Immurium/St. Margarethen in Salzburg
}

Infolge der im Jahre 2005 vorgenommenen Planierungsmaßnahmen auf den Flurstücken westlich des sog. Schindergrabens (Felder des Bauerngutes Ötzengraber) wurden antike Oberflächenfunde getätigt. Die Fundmeldung enthielt weiteres Fundmaterial (Kat. 1-11), das im Zuge der Begehungen von den Abhängen unterhalb der antiken Siedlung zu beiden Seiten des \Schindergrabens` aufgelesen worden war. Sämtliche Funde und Mauerreste wurden von K. Löcker (Archeoprospections ${ }^{\circledR}$ ZAMG, Wien) und Mag. K. Heitzmann (Lungauer Museumsverein Tamsweg) eingemessen und der Salzburger Landesarchäologie gemeldet. Beiden sei für die Überlassung der Planunterlagen und des Materials herzlich gedankt.

Ergänzend werden hier noch Oberflächenfunde (Kat. 12-47) angeschlossen, die ebenfalls im Jahr 2005 im Bereich der Bauten C-I des Vicus aufgelesen wurden ${ }^{1}$.

\section{Fundmünzen}

Neben rezenten Münzen und einer spätmittelalterlichen Silbermünze aus Tirol (Kat. 30) ergänzen Emissionen der Kaiser Gordian III (238-244 n. Chr.) (Kat. 50), Decius (249-251 n. Chr.) (Kat. 26), Trebonianus Gallus (251-253 n. Chr.) (Kat. 27) und Carus (282-283 n. Chr.) (Kat. 28) die Liste der bereits aus Immurium bekannten Gepräge der Soldatenkaiser und der Tetrarchie (Maximinianus [?]) (Kat. 29) ${ }^{2}$. Eine Emission des Kaisers Gratian (Kat. 3) dehnt das Fundmünzenspektrum bis in das letzte Viertel des 4. Jahrhunderts n. Chr. aus.

R. Kastler

Die Fälschung einer Fälschung aus der Zeit um 200 n. Chr. - die Gussmünze (Kat. 51 Abb. 1)

In der Zeit zwischen etwa 180 und $230 \mathrm{n}$. Chr. gelang es der römischen Finanzverwaltung nicht mehr, für die westlichen Donauprovinzen ausreichenden Nachschub an Kleingeld zur Deckung der alltäglichen Geschäfte sicherzustellen. Die Bevölkerung der betroffenen Gebiete griff in der Folge zur Selbsthilfe und stellte in Eigenregie `Kleingeld in beträchtlichen Mengen her. Im Wesentlichen gelangten dabei zwei unterschiedliche Techniken zum Einsatz: einerseits der Nachguss offizieller, geprägter Münzen, andererseits das Prägen von Eisenmünzen mit einem dünnen Kupferüberzug aus selbstgeschnittenen Stempeln in charakteristisch unbe-

\footnotetext{
${ }^{1}$ Grundsätzlich werden im Folgenden lediglich die antiken Fundstücke vorgelegt. Neuzeitliche und mittelalterliche Funde finden nur in Ausnahmefällen Erwähnung. - Die Zitate dieses Beitrags richten sich nach den Richtlinien des Österreichischen Archäologischen Instituts, s. $<$ http://www.oeai.at/publik/auotren.hmtl>, zusätzlich verwendete Kurzzitate finden sich in der Bibliographie am Ende des Beitrags aufgelöst.

2 Moucka-Weitzel 1998, 173-174.
} 
holfenem Stil. Gelegentlich wurden letztere, sog. barbarisierte Prägungen, wiederum von den Werkstätten der Gussfälscher als Vorbilder zum Abgießen genommen. Um eine solche Fälschung einer Fälschung, also einen Guss nach einer Prägefälschung, handelt es sich hier.

M. Pfisterer

\section{Funde aus Bronze}

Trachtzubehör

Im Zuge der 1964-1970 laufenden Ausgrabungen waren lediglich fünf Fibeln ${ }^{3}$ gefunden worden. Unter den Neufunden zählen die Fibeln Kat. 32 und 31 zu den ältesten Typen.

Mit Ausnahme der Nadel und von Ausbrüchen im Bereich des Nadelhalters komplett erhalten ist die kräftig profilierte Fibel mit Kopfplatte und dreifach gelochtem, trapezoidem Nadelhalter Kat. 31 (Abb. 2). Anhand seiner Eingliedrigkeit ist das Stück noch der frühen Gruppe unter den Fibeln der Form Almgren 73, Jobst 4D, Riha 2.9.3, d. h. der Zeit zwischen dem dritten Drittel des 1. Jahrhunderts bis zum Anfang des 2. Jahrhunderts n. Chr. zuzuordnen ${ }^{4}$.

Das Fußfragment Kat. 32 entspricht anhand der Form des Nadelhalters und des Fußes nur allgemein der Typengruppe der kräftig profilierten Fibeln Almgren 70 und 73. Die hochrechteckige Form des Nadelhalters weist hierbei in das 2. Jahrhundert n. Chr. ${ }^{5}$.

Die bis auf die Nadel vollständige Kniefibel Kat. 33 (Abb. 3) weist einen bandförmigem Bügel mit Mittelwulst und volutenartig hochgezogenem Kopf (Sporn) auf. Der kantige, annähernd quadratische Fuß mit rechteckigem Fußknopf ist mit einem schmalen, längs gestellten Nadelhalter ausgestattet. Wolfszahndekor verziert den Ansatz des Mittelwulstes und den Beginn der schmalen, kantigen Kopfplatte. Die Fibel entspricht der Kniefibel mit Spiralhülse Variante Jobst 12 G, einer in Noricum eher seltenen Übergangsform zu den Kniefibeln mit Kopfplatte aus dem späten 2. und der ersten Hälfte des 3. Jahrhunderts n. Chr. ${ }^{6}$.

Anhand des erhabenen Randes (Rahmen), der Reste von Weißmetallüberzug aufweist, ist die Scheibenfibel Kat. 34 (Abb. 4) dem Typus Böhme 44a bzw. Mackensen Var. 1 zuzuweisen. Ein etwaig vorhandener Pressblechdekor im Zentrum ist bei diesem Exemplar verloren. Rahmenscheibenfibeln dieser Art sind vor allem im obergermanisch-rätischen Limesgebiet in der zweiten Hälfte des 2. bis zur Mitte des 3. Jahrhunderts verbreitet ${ }^{7}$.

\section{Zaumzeug und Beschläge}

Pferdegeschirr war bisher unter den Funden aus Immurium lediglich durch eine Zügelzwinge aus Bronze sowie eine Melonenperle und ein Phallusamulett vertreten ${ }^{8}$.

Unter den Neufunden ragt ein nach Aussage des Finders in der vorliegenden Kombination angetroffenes Ensemble mit Pferdegeschirr (Kat. 4) hervor. Es handelt sich um ein kreuzförmiges Backenstück einer Trense (psalion), auf deren einem Knebelarm im Fundzustand ein linsenförmiger Riemenaufschub steckte. Das Seitenstück der Trense (Kat. 4a Abb. 5) besteht aus einem 6,47 cm langen Knebel, in dessen Mitte zwei gegenständige Ösen angebracht sind. Der im Querschnitt halbrunde Knebel ist an den gerade abgeschnittenen Enden 1,03 cm breit und verjüngt sich zur Mitte hin. Der zur Aufnahme der Trense bestimmten Ringöse (Innendurchmesser 1,85 cm) gegenüber befindet sich eine Öse in Form eines gleichschenkeligen

\footnotetext{
${ }^{3}$ Moucka-Weitzel 1998, 160 Taf. 89, 1-5.

${ }^{4}$ Vgl. Jobst 1979, 74 f. Taf. 9, 242 f.; Sedlmayer 1995, 18-21. 134 Taf. 3, 23-25. 28; Ortisi 2002, 24 Taf. 9, 117-120.

${ }^{5}$ Vgl. Jobst 1979, 30 f. Taf. 1-6, 7-46; Riha 1979, 79 f. Taf. 11, 271-277; Sedlmayer 1995, 21 f.; Ortisi 2002, 24.

${ }^{6}$ Vgl. Jobst 1979, 63 Taf. 17, 121; 18, 122. - Ähnlich jedoch Typ Jobst 12 C: Ortisi 2002, 36 Taf. 18, 295.

7 Mackensen 1973, 65 f. Taf. 5, bes. Nr. 2; Ortisi 2002, 41 f. Taf. 22, 389; Sedlmayer 1995, 63 f. Taf. 19, 137.

${ }^{8}$ Moucka-Weitzel 1998, 154. 162 Taf. 87, 7; 89, 7. 24.
} 

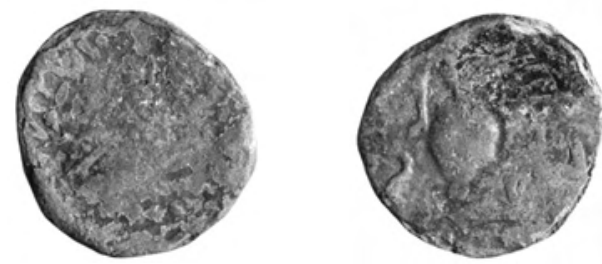

1 Gussmünze Kat. 51, Avers und Revers, M. $1: 1$

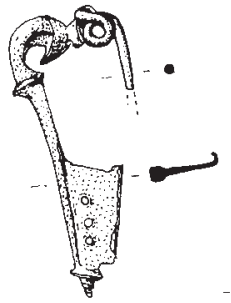

2 Fibel Kat. 31, M. $1: 2$
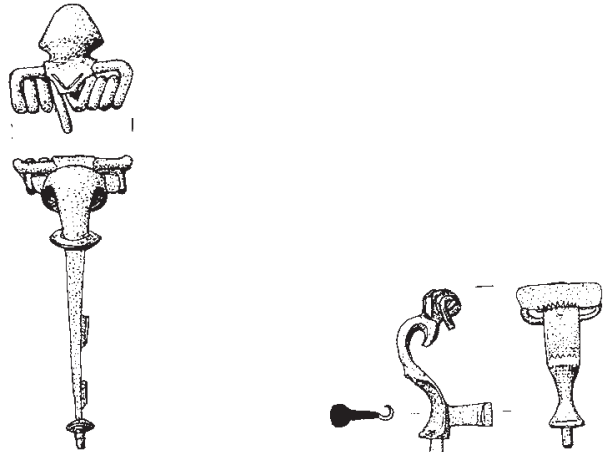

3 Fibel Kat. 33, M. $1: 2$

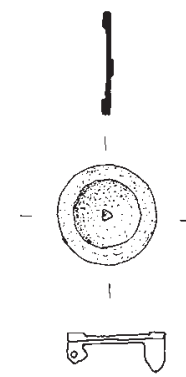

4 Fibel Kat. 34, M. $1: 2$

Dreiecks (größte B 3,2 cm), die zur Aufnahme des Backenriemens bestimmt war. Die engste Parallele zu diesem Fundstück bietet eine Trense aus dem Mitte des 2. bis Mitte des 3. Jahrhunderts n. Chr. belegten Numeruskastell Feldberg im Taunus, bei der allerdings die Knebel S-förmig geschwungen sind. Ein weiteres, jedoch stärker verziertes Objekt ist aus dem Waffenfund der 255/256 n. Chr. zerstörten Befestigungen von Dura Europos ${ }^{9}$ bekannt. Das Trensenseitenstück gehört funktionell zu einer Gruppe meist scheiben- oder dreipassförmiger Backenstücke aus Bronze, die sowohl mit gebrochenen Trensen als auch Stangen- und Hebelstangenbissen kombiniert werden konnte ${ }^{10}$.

Der in Kombination mit dem Trensenteil gefundene, linsenförmige Riemenaufschub (Kat. 4b Abb. 6.) ist auf der Oberseite durch mehrere längs einer Mittelrille verlaufende, parallele Linien dekoriert. Der im Querschnitt halbrund gewölbte Schuber ist auf der Unterseite mit einem rechteckigen Bügel von $2 \mathrm{~cm}$ Länge und $0,7 \mathrm{~cm}$ Breite ausgestattet. Derartige Riemenaufschübe waren Teil der Zügel und verhinderten, neben ihrer Schmuckwirkung, ein Rutschen der Zügelriemen in der Hand des Reiters. Geläufig sind diese Zügelaufschübe in rechteckiger Form mit kräftig profiliertem Rillendekor ${ }^{11}$. Die engste Parallele zu dem Schuber aus Immurium bilden linsenförmige Zügelbeschläge aus dem Wagengrab von Környe und aus Dura Europos ${ }^{12}$. Anhand der Datierung dieser Parallelen in das 3. Jahrhundert n. Chr. ist eine originäre Zugehörigkeit zum Trensenknebel Kat. 4a nicht unmöglich.

Ebenfalls in den Bereich der Zaumzeugbeschläge ist ein Riemenbeschlag (phalera) mit gewölbter Kopfplatte (Kat. 35 Abb. 7) zu zählen. Auf der Unterseite der gewölbten Kreisscheibe sind zwei Ledernieten mitgegossen, von denen eine ausgerissen ist. Dieser Beschlagstyp wird von M. Gschwind als von den Drei-

\footnotetext{
9 Schleiermacher 2000, 169 Taf. 5, 18. - Zur Datierung vgl. Baatz - Herrmann 1982, 266-269; James 2004, 68. 96 Abb. 44 , 327. 328.

${ }^{10}$ Ritterling 1912, 166-168 bes. Taf. 19, 12 f.; Junkelmann 1992, 18 Abb. 3. 12; Vanden Berghe 1996, 89-91; Unz - Deschler-Erb 1997, 50 f. Taf. 68, 1054-1060; Simpson 2000, Taf. 27, 2; Radman-Livaja 2004, 115 Taf. 71 Nr. 513 f.

11 Oldenstein 1976, 174 f. Taf. 51, 588-590; Deimel 1987, 393 Taf. 110, 5; Gschwind 1998, 113 f. Abb. 1, 6-10; Radman-Livaja 2004, 118 Taf. 74, 540; Höck 2006, 255 Abb. 2, 10.

12 Gschwind 1998, 126 Abb. 8, 6; James 2004, 68 Abb. 45, 349.
} 


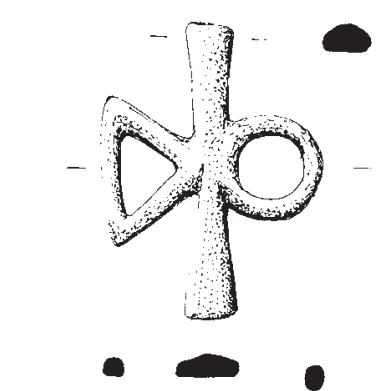

5 Trensenknebel Kat. 4a, M. $1: 2$
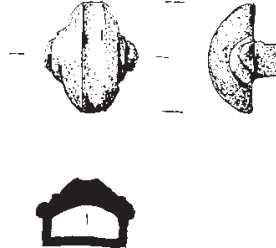

1

6 Zügelaufschub Kat. 4b, M. $1: 2$
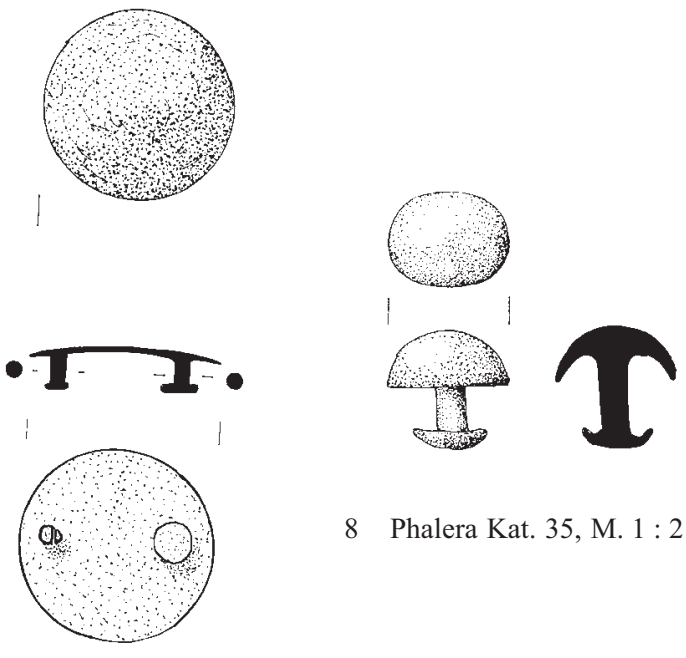

8 Phalera Kat. 35, M. $1: 2$

7 Phalera Kat. 35, M. $1: 2$

Biger- und Vierzigerjahren des 3. Jahrhunderts n. Chr. bis in die Anfangsjahre des 4. Jahrhunderts n. Chr. als gebräuchlich angesehen ${ }^{13}$.

Anhand des großen Durchmessers könnte wohl auch der Beschlag mit pilzförmiger Gegenplatte (Kat. 36 Abb. 8) zum Bestand an Pferdegeschirr zählen, obwohl derartige Beschläge auch bei den Ringschnallen (cingula) Verwendung fanden. Aufgrund des Gegenknopfes zählt der Beschlag zu der frühestens nach der Mitte des 2. und im 3. Jahrhundert n. Chr. gebräuchlichen Form ${ }^{14}$.

Weniger präzise funktionell zuzuweisen ist ein Beschlag mit flacher Kopfplatte mit angegossenem rundem Gegenniet in Bronze (Kat. 37). Möglicherweise handelt es sich bei diesem Objekt um ein Bruchstück eines größeren Beschlags ${ }^{15}$.

\section{Geräte}

Im Zusammenhang mit dem Pferdegeschirr könnte auch eine stark deformierte Bronzeglocke (Kat. 38, Typ B2 nach Nowakowski) stehen ${ }^{16}$.

Ebenfalls zu den Gerätschaften sind zwei Schlüssel aus Bronze zu zählen. Der kleine Schiebeschlüssel (Kat. 5 Abb. 9) vom Typ mit unterständigen Zinken und einfach abgewinkeltem Bartsteg und zwei Zinken scheint eine Zwischenform der Schlüssel mit Ringende und der eigentlichen Ringschlüssel darzustellen ${ }^{17}$. Ringschlüssel werden aufgrund ihrer kleinen Dimension Kästchen und kleinen Behältern zugeordnet. Ob es sich dabei zwingend immer um die weibliche Besitzsphäre handeln muss, sei dahingestellt ${ }^{18}$. Der größere Schiebeschlüssel (Kat. 6 Abb. 10) mit dreieckiger Ringschulter (Typ Schütz Griff V, Schaft I, Querschnitt I Bart I $)^{19}$ weist auf der Rückseite deutliche Feilspuren und eine Gusskehlung vom Herstellungsprozess auf.

\footnotetext{
13 Oldenstein 1976, 186 f. Taf. 56, 686-695; Gschwind 1998, 122-124; Schleiermacher 2000, $167-193$ bes. 190 f. Taf. 9; James 2004, 69. 95 Abb. 314. 315.

14 Oldenstein 1976, 167 f. Taf. 46, 485-489; James 2004, 60 f. Abb. 31 a, 91; Abb. 42, 283. 286 f.; Bishop - Coulston 2006 , 182 f. Abb. 118, 12. Zum Pferdegeschirr: Gschwind 1998, 115 f. Abb. 2, 2-4; Schleiermacher 2000, Taf. 6, $32-34$.

15 Derartige Beschläge Oldenstein 1976, 171 Taf. 47 f., 512-527.

16 Flügel 1993, 99. 101 Taf. 34, 179. Zur Verwendung bei Pferdegeschirr Junkelmann 1992, 15 Abb. 4 ; S. 85.

17 Schütz 2003, 97 f. zur Typologie; Vergleichsstück Deimel 1987, 57. 187 Taf. 38, 11; Moucka-Weitzel 1998 , 164 Abb. 53.

18 Schütz 2003, $113 \mathrm{f}$.

19 Schütz 2003, 102 Abb. 40.
} 


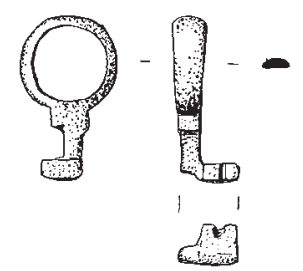

9 Schlüssel Kat. 5, M. $1: 2$

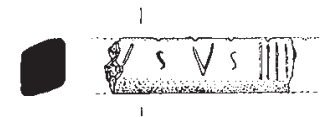

11 Waagbalken Kat. 40, M. $1: 2$
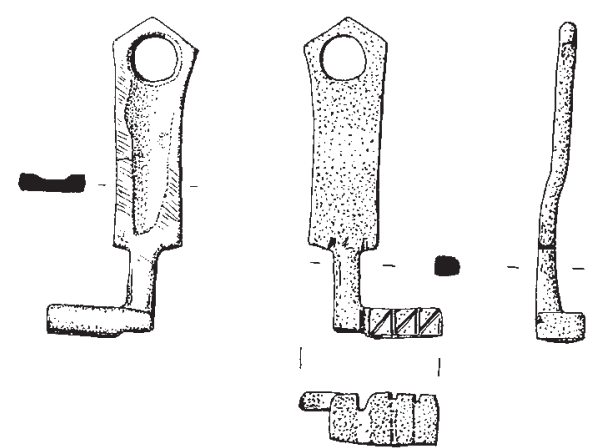

10 Schlüssel Kat. 6, M. $1: 2$

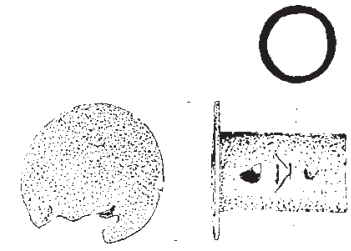

12 Waagbalkenbeschlag Kat. 41, M. $1: 2$

Möglicherweise ist das verbrannte Fundstück Kat. 39 in diese Gruppe einzureihen. Es handelt sich dabei um eine ehemals rechteckige Bronzetülle, die durch Hitzeeinwirkung stark verschmolzen ist: Am oberen Ende ist eine verschmolzene Doppelwulstverzierung erkennbar. Im Inneren der Tülle sind noch Eisenreste erhalten. Eventuell handelt es sich bei diesem Fundstück um den Bronzegriff eines Schlüssels ${ }^{20}$.

Bereits im Zuge der Ausgrabungen 1964-1970 war ein Waagbalken einer Schnellwaage vom Typ Pompeji gefunden worden ${ }^{21}$. Unter den Neufunden ist das Bronzebruchstück Kat. 40 (Abb. 11) einer Schnellwaage zuzuweisen. Der im Querschnitt rhombische Waagbalken weist auf der Oberkante vier Kerben auf, die mit der von rechts nach links laufenden Beschriftung: IIII V V[I] für das Gewicht in librae korrespondieren. S-förmige Gravuren dienen dabei als Kennzeichen für semis, d. h. halbe librae ${ }^{22}$. Die Unterseite des Gewichtarms mit Messskala trägt eine flache Kerbe.

Möglicherweise ebenfalls um die Endtülle eines hölzernen Gewichtarms einer Schnellwange handelt es sich bei Kat. 41 (Abb. 12). Die der Tülle einer Schnellwaage von Mauer an der Url entsprechende Gestaltung des Fundstücks und der Innendurchmesser von 1,4 cm sprechen für eine derartige Zuweisung ${ }^{23}$. Der auf der Tülle erkennbare Buchstabe ist als Rest der Skalengliederung zu deuten.

\footnotetext{
${ }^{20}$ Deimel 1987, 183 f. Taf. 38, bes. 1-4.

${ }^{21}$ Moucka-Weitzel 1998, 163 Taf. 90, 11. Zum Typ: Franken 1993, 69-120 bes. 77-81; Grönke - Weinlich 1992, 189-230 bes. $194 \mathrm{zu}$ semis.

${ }^{22}$ Franken 1995, 425 f.; 428 Kat. B3.3.

${ }_{23}$ Garbsch 1992, 231-259 bes. 236 Abb. 2; Garbsch 1994, 275-282. Zum Typ: Franken 1993, 100-102.
} 
Unbestimmte Bronzefunde

Während sich ein Blechabschnitt (Kat. 43) mit deutlichen Treibspuren wohl als Teil eines zur Materialgewinnung genutzten Gefäßes deuten lässt, kann der mit drei parallelen Rippen und Kreispunzen verzierte Blechabschnitt Kat. 44 nicht näher bestimmt werden. Auch ein im Querschnitt halbrunder Ring (Kat. 42) erlaubt keine nähere Funktionsbestimmung.

\section{Funde aus Blei}

Neben zahlreichen rezenten Bleikugeln ist in dieser Materialgruppe besonders ein trapezförmiges Bleietikett mit im Bereich der Lochung (Dm 0,36 $\mathrm{cm}$ ) halbrund auslaufender Oberseite (Kat. 7) zu nennen. In Form und Gestaltung entspricht es dem bereits während der Grabungen 1964-1970 aufgefundenen Attegenta-Etikett ${ }^{24}$. Reste einer Beschriftung sind jedoch nicht zu erkennen. Ein weiteres, annähernd quadratisches Bleistück (Kat. 45) könnte ebenfalls von einem Etikett stammen.

Ferner ist unter den Bleifunden ein trapezförmiges Werkstück zu nennen, möglicherweise ein Barren (Kat. 8 Abb. 13). Das im Querschnitt flach-rechteckige Blei läuft nach oben hin stangenförmig-rund aus. In diesem Bereich sind deutliche Spuren einer von einer Zange (?) verursachten Quetschung zu erkennen.

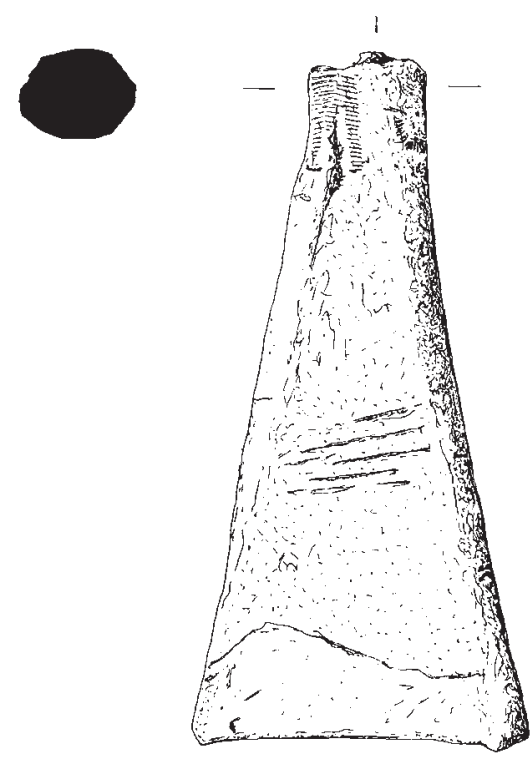

13 Bleibarren Kat. 8, M. $1: 2$

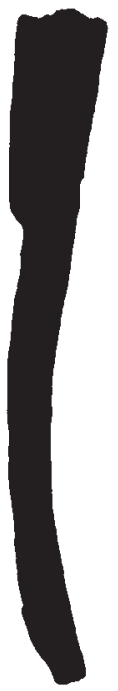

\section{Funde aus Eisen}

Möglicherweise antik ist der scheibenförmige Beschlag mit zentraler rechteckiger Ausnehmung (Kat. 46). Es könnte sich um den Beschlag eines Türziehers handeln. Unter den Funden des Kärntner Magdalensberges sind jedoch derartige schüsselförmige Bleche nicht vertreten ${ }^{25}$.

\section{Funde aus Glas}

Die kleine, massive Mündung mit kurzem Hals und fallender Schulter stammt von einer kleinen Glasflasche (Kat. 9), die in das 1. Jahrhundert n. Chr. datiert werden kann ${ }^{26}$.

Die mit einem Gittermuster aus Glasfäden verzierte Wandung (Kat. 10) dürfte von einem Becher oder einem geschlossenen, bauchigen Gefäß mit netzförmigem Glasfadendekor stammen, wie sie im 3. Jahrhundert n. Chr. bis zum Beginn des folgenden Jahrhunderts verbreitet sind. Der deutlich durch die Glaswandung geschmolzene Faden, die Dimension der Gefäßrundung und die gleichmäßige Gitterstruktur passen eher dazu, als zu einem optisch verwandten, allerdings deutlich älteren Knospenbecher, dessen Netzmuster durch eine Form erzeugt wurde ${ }^{27}$. Vergleichbare Stücke wurden bereits 1964-1970 im Bereich von Haus J gefunden ${ }^{28}$.

${ }^{24}$ Fleischer 1998, 48 Abb. 49.

${ }^{25}$ Künzl 2003, 242 Abb. 33; Schütz 2003, 149-158.

${ }^{26}$ Vgl. Barkóczi 1988, 110 Taf. 16, 184. 186.

${ }^{27}$ Ad Knospenbecher: Rütti 1988, 36 Taf. 8. 38 Nr. 664 f. Typ Berger Variante c. Gefäße mit Netzdekor aus Glasstegen: Fremersdorf 1959, 73 f. Taf. 114-117; Follmann-Schultz 1992, 52 Nr. 29.

${ }^{28}$ Moucka-Weitzel 1998, 156 Taf. 87, 21. 


\section{Baukeramik}

Ebenfalls aufgelesen wurde das Bruchstück eines tubulus (Kat. 11) mit Kreuzschraffur für die Putzhaftung.

\section{Zusammenfassung}

Obwohl die vorgelegten Funde nicht stratifiziert sind, sondern lediglich aus Oberflächenaufsammlungen stammen, besitzen sie durchaus Aussagekraft für die Topographie und Geschichte Immuriums.

Die aus dem Bereich des sog. Ötzgraberfeldes gemeldeten Funde dürften als Hinweise auf die römerzeitliche Datierung der prospektierten Mauerstruktur gelten. Die Neufunde von Waagbalken und Bleietiketten ergänzen die Interpretation des zur Straßenstation gehörenden vicus Immurium als wichtigem Zwischenhandelsort südlich des Radstädter Tauerns.

Eine augenfällige Ergänzung des Fundspektrums stellen jedoch die Bestandteile von Pferdegeschirr dar. Grundsätzlich verwundern solche Funde im Rahmen einer auf die Verkehrsinfrastruktur ausgerichteten Fundstelle wohl kaum, erstaunlich hingegen ist ihr chronologischer Aspekt: Die Funde lassen sich weitestgehend dem entwickelten 2. und 3. Jahrhundert n. Chr. zuweisen, deutlich frührömische Pferdegeschirrbeschläge hingegen fehlen. Dieser zeitliche Ausschlag dürfte jedoch, betrachtet man das übrige Fundspektrum und besonders die chronologische Verteilung der Münzfunde, mit der allgemeinen Entwicklung von Immurium übereinstimmen. Bedingt durch den Ausbau der Tauernroute unter den Severern und mangels Auswirkungen der Markomannenkriege zeigt sich bei den Fundmünzen ein deutliche Mengenkonzentration, mit Schwerpunkt im 2. und 3. Jahrhundert n. Chr., wobei für die Zeit der Soldatenkaiser eine grundsätzlich höhere Emissionsrate zu berücksichtigen wäre.

Die Pferdegeschirrbeschläge sind demnach ebenfalls als Zeugnisse der intensiven Straßennutzung zu werten. Die Frage ist allerdings, durch wen, da diese Objekte meist durch Vergleiche aus militärischem Umfeld bekannt sind. Wie bereits M. Mackensen dargelegt hat, ist ohne einen gesicherten militärischen Kontext bei Funden von Pferdegeschirr durchaus auch von einer Nutzung im zivilen Umfeld auszugehen ${ }^{29}$ im Bereich einer Straßenstation den Erwartungen entsprechend. Dennoch darf, entsprechend der Wertung von Militariafunden aus Immurium durch R. Fleischer, auch gerade für eine Straßenstation die Präsenz von Militär als Reisende oder gar als temporär stationierte Benefiziarier nicht ausgeschlossen werden ${ }^{30}$.

Hinzugefügt zum Bestand der Militaria muss in dieser Hinsicht auch die bereits unter den Altfunden 1964-1970 gelistete Zierplatte ${ }^{31}$ aus Bronze werden. Bei dieser 4,8 cm großen Zierscheibe handelt es sich um einen Balteusschließe. Mit dem späteren 2. und 3. Jahrhundert n. Chr. wurde parallel zur Einführung der spatha für alle Truppenteile auch die Trageweise geändert. Der über die Schulter getragene Schwertgurt balteus ersetzte die bisherige cingulum-Gürtung. Zum Verschluss des Schultergurtes diente eine Bronzescheibe, auf deren Rückseite eine rechteckige Öse angebracht war. Diese ist bei dem Stück aus Immurium ausgebrochen. Der Balteusbeschlag aus Immurium gehört zur Gruppe der durchbrochenen Beschläge ${ }^{32}$. Sein swastikartiges Motiv findet dabei unter den Funden von Dura Europos seine engste Entsprechung und belegt damit auch für das 3. Jahrhundert n. Chr. eine Militärpräsenz unbestimmter Dauer ${ }^{33}$.

R. Kastler

\footnotetext{
${ }^{29}$ Mackensen 2001, 325-346 bes. 336-342.

${ }^{30}$ Fleischer 1998, 49 f. Die bei Fleischer 1998, 172 Taf. 96, 16-23 aufgelisteten Pfeil- und Speerspitzen wurden von der Bearbeiterin Moucka-Weitzel 1998, 172 zu Recht als mittelalterlich angesprochen; vgl. Stadler 1995, 248 f. Von den Immurium-Funden bei Moucka-Weitzel 1998, 172 könnten die Pfeilspitze Taf. 96, 20 und die Bolzen Taf. 97, 5 f. antik sein. - Vgl. Radman-Livaja 2004, 57 Taf. 167, 68 zu Moucka-Weitzel 1998, Taf. 96, 20; Radman-Livaja 2004, 58 Taf. 169, 89 zu Moucka-Weitzel 1998, Taf. 97, 5; James 2004, 218 Abb. 130, 790 zu Moucka-Weitzel 1998, Taf. 96, 20 und James 2004, Abb. 130, 800. 803 zu MouckaWeitzel 1998, Taf. 97, 5 f.

${ }^{31}$ Moucka-Weitzel 1998, 161 Taf. 89, 8.

${ }_{32}$ Zum Typ: Bishop - Coulston 2006, 154-163 bes. 162 Abb. 94. 100; Oldenstein 1976, 226-234 Taf. 84-86.

${ }^{33}$ James 2004, 62. 74 Abb. 36, 26.
} 


\title{
Katalog der Fundstücke
}

\author{
Fundaufsammlung `Schindergraben $`$ - Ötzgraberfeld
}

\section{Münzliste}

Kat. 1

Nominal: $\mathrm{S}$

FO: Bereich des ehemaligen Schwimmbeckens unterhalb des Mithräums

AO: Lungauer Heimatmuseum Tamsweg, o. Inv.

Prägeherr: Commodus (180-192 n. Chr.) für Crispina

RS Legende/Bild: C]ONCO [RDIA] S C; Concordia, sitzend nach links, hält Patera und Cornucopiae

Münzstätte: Rom

Besonderheiten: abgegriffen

Münzzeichen:

Referenz: RIC 665

Prägeperiode: vor $183 \mathrm{n}$. Chr.

Stempelstellung: $26.12 \mathrm{~g} 12^{\mathrm{h}}$

Kat. 2

ohne Abb.

Nominal: Ant

FO: Bereich des ehemaligen Schwimmbeckens unterhalb des Mithräums

AO: Lungauer Heimatmuseum Tamsweg, o. Inv.

Prägeherr: Probus (276-282 n. Chr.)

RS Legende/Bild: RESTITVTORBIS; Frau nach rechts reicht

Kranz an den mit Globus und Szepter stehenden Kaiser

Münzstätte: Sisica

Besonderheiten: stark korrodiert

Münzzeichen:

$$
\underline{\text { XX IB }}
$$

Referenz: RIC 733

Prägeperiode: undatiert

Stempelstellung: 15

Kat. 3

ohne Abb.

Nominal: Follis

FO: Bereich des ehemaligen Schwimmbeckens unterhalb des Mithräums

AO: Lungauer Heimatmuseum Tamsweg, o. Inv.

Prägeherr: Gratian (367-383 n. Chr.)

RS Legende/Bild: GLORIA ROMANORVUM; Kaiser nach rechts vorgehend, zieht mit Rechter Gefangenen am Schopf, in Linker Labarum

Münzstätte: Sisica

Besonderheiten: -

Münzzeichen:

$$
\frac{\mathrm{M} \mid * \mathrm{P}}{\Gamma \mathrm{SISC}}
$$

Referenz: RIC 14c

Prägeperiode: $367-375 \mathrm{n}$. Chr.

Stempelstellung: $11: 30^{\mathrm{h}}$

\section{Fundliste}

Kat. 4

Abb. 5. 6

Seitenstück (Riemenverteiler) einer Trense mit Zügelschuber, Bronze

FO: Ötzgraberfeld

AO: Lungauer Heimatmuseum Tamsweg, o. Inv.

Riemenschuber L 6,47 cm, B 4,86 cm; Beschlag L 2,39 cm, B $2,15 \mathrm{~cm}, \mathrm{H} 1,43 \mathrm{~cm}$

Kat. 5

Abb. 9

Schlüssel mit ringförmigem Griff, Bronze

FO: Ötzgraberfeld

AO: Lungauer Heimatmuseum Tamsweg, o. Inv

L 3,61 cm, Dm 2,15 cm

Kat. 6

Abb. 10

Schlüssel eines Schubschlosses, Bronze

FO: Ötzgraberfeld

AO: Lungauer Heimatmuseum Tamsweg, o. Inv.

L $6,98 \mathrm{~cm}, \mathrm{~B} 3,3 \mathrm{~cm}$

Kat. 7

Etikett, Blei

ohne Abb.

FO: >Schindergraben

AO: Lungauer Heimatmuseum Tamsweg, o. Inv.

L 3,18 cm, B 2,91 cm, Dm 0,52 cm

Kat. 8

Abb. 13

Barren (?), Blei

FO: >Schindergraben

AO: Lungauer Heimatmuseum Tamsweg, o. Inv.

L $14,78 \mathrm{~cm}$, B $7,26 \mathrm{~cm}$, Dm oben $0,73 \mathrm{~cm}$, Dm unten $2,55 \mathrm{~cm}$

Kat. 9

ohne Abb.

Flaschenmündung, Glas

FO: Ötzgraberfeld

AO: Lungauer Heimatmuseum Tamsweg, o. Inv.

Dm $1,64 \mathrm{~cm}$

Kat. 10

ohne Abb.

Wandfragment, Glas

FO: Ötzgraberfeld

AO: Lungauer Heimatmuseum Tamsweg, o. Inv. erhaltene L 3,57 cm; erhaltene B 2,96 cm

Kat. 11

ohne Abb.

Fragment eines Heizziegels, Ton

FO: Ötzgraberfeld

M. Pfisterer erhaltene L $21,3 \mathrm{~cm}$, erhaltene B $10,8 \mathrm{~cm}$ 


\section{Fundaufsammlung >Wilczekfelder}

\section{Münzliste}

Kat. 12

Nominal: $\mathrm{S}$

AO: Salzburg Museum Inv. 17/005

Prägeherr: kaiserzeitlich - Vespasian (?)

RS Legende/Bild: -

Münzstätte: Rom

Besonderheiten: völlig abgegriffen und korrodiert, verbrannt

Münzzeichen: -

Referenz: -

Prägeperiode: -

Stempelstellung: 25g

\section{Kat. 13}

Nominal: Dp

AO: Salzburg Museum Inv. 7/005

Prägeherr: Trajan (98-117 n. Chr.)

RS Legende/Bild: [TR P COS II PP - SC]; Abundantia, Szepter haltend und nach links sitzend, auf einem Sessel, der aus zwei Cornucopiae geformt ist.

Münzstätte: Rom

Besonderheiten: stark abgegriffen, VS Oberfläche in Legende stark abgesplittert, RS stark korrodiert und abgeschliffen, unbestimmt.

Münzzeichen: -

Referenz: RIC 382 Typ

Prägeperiode: $98-103$ n. Chr.

Stempelstellung: $10 \mathrm{~g} 6^{\mathrm{h}}$

\section{Kat. 14}

ohne Abb.

Nominal: S

AO: Salzburg Museum Inv. 12/005

Prägeherr: Hadrian (?)

RS Legende/Bild: sitzende Figur, rechter Arm erhoben mit Figur oder Objekt

Münzstätte: Rom

Besonderheiten: Oberfläche teilweise bis zum Kern korrodiert, unbestimmt

Münzzeichen: -

Referenz: -

Prägeperiode: -

Stempelstellung: $21 \mathrm{~g} 6^{\mathrm{h}}$

\section{Kat. 15}

ohne Abb.

\section{Nominal: S}

AO: Salzburg Museum Inv. 11/005

Prägeherr: Antoninus Pius (?)

RS Legende/Bild: Thronende Gestalt, unbestimmt

Münzstätte: Rom

Besonderheiten: Oberfläche teilweise bis zum Kern korrodiert, unbestimmt

Münzzeichen: -

Referenz: -

Prägeperiode: -

Stempelstellung: $19 \mathrm{~g} 12^{\mathrm{h}}$

\section{Kat. 16}

ohne Abb.

Nominal: As

AO: Salzburg Museum Inv. 9/005

Prägeherr: wohl kaiserzeitlich

RS Legende/Bild: -

Münzstätte: Rom (?)

Besonderheiten: Oberfläche teilweise bis zum Kern korrodiert, unbestimmt

Münzzeichen: -

Referenz: -

Prägeperiode: -

Stempelstellung: $4 \mathrm{~g} 6^{\mathrm{h}}$

Kat. 17

ohne Abb.

Nominal: As

AO: Salzburg Museum Inv. 10/005

Prägeherr: wohl kaiserzeitlich

RS Legende/Bild: -

Münzstätte: Rom

Besonderheiten: Oberfläche teilweise bis zum Kern korrodiert, unbestimmt

Münzzeichen: -

Referenz: -

Prägeperiode: -

Stempelstellung: $5 \mathrm{~g}$

\section{Kat. 18}

ohne Abb.

Nominal: Dp/As

AO: Salzburg Museum Inv. 13/005

Prägeherr: wohl kaiserzeitlich

RS Legende/Bild: -

Münzstätte: Rom

Besonderheiten: völlig abgegriffen und korrodiert, unbestimmt

Münzzeichen: -

Referenz: -

Prägeperiode: -

Stempelstellung: $14 \mathrm{~g}$

Kat. 19

ohne Abb.

Nominal: As

AO: Salzburg Museum Inv. 14/005

Prägeherr: Hadrian (117-138 n. Chr.)

RS Legende/Bild: sitzende weibliche Figur auf Thron mit ausgestrecktem rechtem Arm

Münzstätte: Rom

Besonderheiten: stark abgegriffen und korrodiert, Büste schemenhaft erkennbar

Münzzeichen: -

Referenz: -

Prägeperiode: -

Stempelstellung: $16 \mathrm{~g}$

Kat. 20

ohne Abb.

Nominal: $\mathrm{S}$

AO: Salzburg Museum Inv. 15/005

Prägeherr: wohl kaiserzeitlich, Büste 
RS Legende/Bild: -

Münzstätte: Rom (?)

Besonderheiten: stark abgegriffen und korrodiert, Büste schemenhaft erkennbar

Münzzeichen: -

Referenz: -

Prägeperiode: -

Stempelstellung: $16 \mathrm{~g}$

\section{Kat. 21}

ohne Abb.

Nominal: Dp

AO: Salzburg Museum Inv. 1/005

Prägeherr: Marc Aurel (161-180 n. Chr.)

RS Legende/Bild: SALVTI AUG Cos III S C; Salus stehend nach links, füttert um Altar geringelte Schlange und hält ein Szepter

Münzstätte: Rom

Besonderheiten: abgegriffen

Münzzeichen: -

Referenz: RIC 982

Prägeperiode: Dezember 169 - Dezember 170 n. Chr. Stempelstellung: $5^{\text {h }}$

Kat. 22

ohne Abb.

Nominal: $\mathrm{S}$

AO: Salzburg Museum Inv. 2/005

Prägeherr: Marc Aurel (161-180 n. Chr.) für Faustina

RS Legende/Bild: [SIDERI]BVS [RECEPTA] S C; Diana, stehend nach rechts, hält eine Fackel; Halbmond hinter dem Nacken

Münzstätte: Rom

Besonderheiten: abgegriffen

Münzzeichen: -

Referenz: RIC 1715

Prägeperiode: -

Stempelstellung: $19 \mathrm{~g} 7^{\mathrm{h}}$

\section{Kat. 23}

ohne Abb.

Nominal: S

AO: Salzburg Museum Inv. 3/005

Prägeherr: anhand Frisur und weiblicher Büste nach links:

Marc Aurel (161-180 n. Chr.) für Faustina

RS Legende/Bild: -

Münzstätte: -

Besonderheiten: abgegriffen und korrodiert, scheinbar verbrannt

Münzzeichen: -

Referenz: -

Prägeperiode: -

Stempelstellung: $9 \mathrm{~g} 6^{\mathrm{h}}$

\section{Kat. 24}

ohne Abb.

Nominal: D

AO: Salzburg Museum Inv. 4/005

Prägeherr: Septimius Severus (193-211 n. Chr.)

RS Legende/Bild: RESTITVTOR VRBIS; Roma auf Schild sitzend nach links hält Palladium und Speer

Münzstätte: Rom

Besonderheiten: abgegriffen, Rand stellenweise eingerissen Münzzeichen: -
Referenz: RIC 288

Prägeperiode: $202-210 \mathrm{n}$. Chr

Stempelstellung: $1^{\mathrm{h}}$

Kat. 25

ohne Abb.

Nominal: S (?)

AO: Salzburg Museum Inv. 16/005

Prägeherr: severerzeitlich

RS Legende/Bild: -

Münzstätte: Rom (?)

Besonderheiten: stark abgegriffen, Büste schemenhaft erkennbar

Münzzeichen: -

Referenz: -

Prägeperiode: -

Stempelstellung: $10 \mathrm{~g} 12^{\mathrm{h}}$

Kat. 26

ohne Abb.

Nominal: Ant

AO: Salzburg Museum Inv. 5/005

Prägeherr: Decius (249-251 n. Chr.)

RS Legende/Bild: GENIVS EXERCILLVRICIANI

Münzstätte: Rom

Besonderheiten: abgegriffen, RS teilweise Doppelprägung

Münzzeichen: -

Referenz: RIC 16c

Prägeperiode: $249-251 \mathrm{n}$. Chr.

Stempelstellung: $6^{\mathrm{h}}$

Kat. 27

ohne Abb.

Nominal: Ant

AO: Salzburg Museum Inv. 6/005

Prägeherr: Trebonianus Gallis (251-253 n. Chr.)

RS Legende/Bild: [L]IBERTAS AVG[G]; Libertas, stehend nach links, hält Pileus und Szepter mit Stern im Feld

Münzstätte: Rom

Besonderheiten: abgegriffen, Schrötling an zwei Seiten abgebrochen

Münzzeichen: -

Referenz: RIC 38

Prägeperiode: -

Stempelstellung: $6^{\text {h }}$

Kat. 28

ohne Abb.

Nominal: Ant

AO: Salzburg Museum Inv. 8/005

Prägeherr: Carus (282-283 n. Chr.)

RS Legende/Bild: IOVI VICTORI; Jupiter, stehend nach rechts, hält Victoria auf Globus und Szepter; Adler zu Füßen Münzstätte: Rom

Besonderheiten: abgegriffen, geringe Reste von Silbersud

Münzzeichen: KAB

Referenz: RIC 39

Prägeperiode: -

Stempelstellung: $12^{\mathrm{h}}$

Kat. 29

ohne Abb.

Nominal: Follis

AO: Salzburg Museum Inv. 18/005

Prägeherr: tetrarchisch, Maximinianus (?) 
RS Legende/Bild: [MONETA SACRA] AVGGE[T CAESS] NN; Moneta, stehend nach links, hält in der Rechten Waagschale, in der Linken Füllhorn

Münzstätte: -

Besonderheiten: völlig abgegriffen und stark korrodiert

Münzzeichen: -

Referenz: -

Prägeperiode: um 300

Stempelstellung: $7 \mathrm{~g} 6^{\mathrm{h}}$

Kat. 30

ohne Abb.

Nominal: Kreuzer

AO: Salzburg Museum Inv. 19/005

Prägeherr: MA Tirol

M. Pfisterer

\section{Sonstige Fundstücke}

Kat. 31

Abb. 2

Eingliedrig kräftig profilierte Fibel mit Kopfplatte, Bronze

AO: Salzburg Museum, o. Inv.

L $6,3 \mathrm{~cm}$

Spirale mit acht Windungen, obere Sehne, trapezförmige Sehnenkappe (leicht ausgebrochen), rechteckige Kopflatte, gestreckter, an Unterseite hochgezogener Kopf, zweifach profilierter, dreiviertelkreisförmiger Bügelknopf, gestreckter Fuß mit rechteckigem Querschnitt, trapezoider, dreifach gelochter Nadelhalter, dreifach profilierter Fußknopf, Nadel abgebrochen, korrodiert.

\section{Kat. 32}

ohne Abb.

Fuß einer kräftig profilierten Fibel, Bronze

AO: Salzburg Museum, o. Inv.

erhaltene L 2,63 cm, B 1,82 cm

Bruchstück des Fußes mit rechteckigem Querschnitt, dreifach profiliertem, im Querschnitt rechteckigem Fußknopf, hoher rechteckiger Nadelhalter, Nadelrast ausgebrochen.

Kat. 33

Abb. 3

Kniefibel mit rechteckiger Kopfplatte, Bronze

AO: Salzburg Museum, Inv. 28/005

L 3,59 cm, B $2 \mathrm{~cm}, \mathrm{H} 1,76 \mathrm{~cm}$

Spirale mit sieben Windungen und unterer Sehne, Nadel fehlt. Bandförmiger Bügel mit Mittelwulst und volutenartig hochgezogenem Kopf (Sporn). Quadratischer Fuß mit rechteckigem Fußknopf. Hoher längsgestellter Nadelhalter. Wolfszahndekor bei Mittelwulst und Kopfplattenansatz.

\section{Kat. 34}

Abb. 4

Rahmenscheibenfibel, Bronze

AO: Salzburg Museum, Inv. 27/005

Dm 2,21 cm, erhaltene H 0,91 cm, 0,29 cm breiter, erhabener Rand mit Resten von Weißmetallüberzug.

Kat. 35

Abb. 7

Riemenbeschlag mit gewölbter Kopfplatte, Bronze AO: Salzburg Museum, Inv. 21/005

Dm $4,11 \mathrm{~cm}$
Kat. 36

Abb. 8

Beschlag mit pilzförmiger Kopfplatte, Bronze

AO: Salzburg Museum, Inv. 22/005

Dm 2,62 cm, H 2,66 cm

Mitte 2.-1. Hälfte 3. Jh.

Kat. 37

ohne Abb.

Beschlag mit flacher Kopfplatte, Bronze

AO: Salzburg Museum, Inv. 23/005

erhaltene B $1,51 \mathrm{~cm}, \mathrm{H} 0,7 \mathrm{~cm}$

Flache, unregelmäßig ausgebrochene Platte mit angegossenem rundem Gegenniet.

\section{Kat. 38}

ohne Abb.

Glocke, Bronze, Eisen

AO: Salzburg Museum, Inv. 20/005

H 8,14 cm, B 4,53 cm

Glockenmantel verquetscht. Eiserne Klöppelhalterung am Ansatz abgebrochen (Klöppel fehlt).

\section{Kat. 39}

ohne Abb.

Schlüsselgriff (?), Bronze, Eisen

AO: Salzburg Museum, o. Inv.

erhaltene L 4,19 cm, B 2,8 cm, Dm 1,45 cm

Rechteckige Bronzetülle (Wandungsstärke 0,22 cm), stark verschmolzen. Reste einer umlaufenden Doppelwulstverzierung erkennbar; im Inneren Eisenreste.

Kat. 40

Abb. 11

Waagbalken mit Skalengliederung, Bronze

AO: Salzburg Museum, Inv. 25/005

erhaltene L $4 \mathrm{~cm}, \mathrm{H} 1,35 \mathrm{~cm}$

\section{Kat. 41}

Abb. 12

Tülle eines hölzernen Waagbalkens (?), Bronze

AO: Salzburg Museum, Inv. 26/005

Dm 3,08 cm, erhaltene H 2,93 cm

Scheibenförmiger Boden (ausgebrochen), an dessen Zentrum eine zylinderförmige Tülle (Dm $1,72 \mathrm{~cm}$, Wandungsstärke $0,11 \mathrm{~cm}$ ) ansetzt. Objekt in einem Guss gefertigt. Die Tülle weist einige Gussfehlstellen, jedoch kein Befestigungsloch auf. 1,3 cm unterhalb der ausgebrochenen Tüllenmündung der (im Wachsmodel?) eingravierte Buchstabe A; oberhalb weiterer Ansatz einer Haste.

Kat. 42

ohne Abb.

Bronzering mit halbrundem Querschnitt $(0,2 \mathrm{~cm})$ stark.

AO: Salzburg Museum, Inv. 24/005

Dm $2,16 \mathrm{~cm}$

Kat. 43

ohne Abb.

Blechabschnitt, Bronze

AO: Salzburg Museum, o. Inv.

erhaltene L $5,84 \mathrm{~cm}$, erhaltene B $2,65 \mathrm{~cm}$

Blech (Stärke $0,11 \mathrm{~cm}$ ) mit deutlichen Treibspuren auf der Innen- und Außenseite. Auf drei Seiten unregelmäßige Bruchschnittkanten. 
Kat. 44

Bruchstück eines Zierblechs, Bronze

AO: Salzburg Museum, o. Inv.

erhaltene L $34 \mathrm{~cm}$, erhaltene B $1,89 \mathrm{~cm}$

Unregelmäßig ausgebrochenes, $0,12 \mathrm{~cm}$ dünnes Bronzeblech, in der Mitte verziert mit drei plastischen Rippen, die wiederum mit vier parallelen Reihen Kreispunzen verziert sind.

\section{Kat. 45}

ohne Abb.

Etikett (?), Blei

AO: Salzburg Museum, o. Inv. erhaltene L 2,95 cm, B 2,32cm

Rechteckiges Bleistück mit geglätteter Oberfläche.

Kat. 46

Türbeschlag, Eisen

AO: Salzburg Museum, Inv. 30/005

Dm $6,79 \mathrm{~cm}$

Scheibenförmiger Beschlag, ehemals schüsselförmig gewölbt, im Zentrum quadratische Ausnehmung von $0,9 \mathrm{~cm}$.

R. Kastler

Fundaufsammlung Ötzgraberfeld

Münzliste

\section{Kat. 47}

Nominal: D

AO: Lungauer Heimatmuseum Tamsweg, o. Inv.

Prägeherr: Septimius Severus (193-211 n. Chr.) SEPT SEV PE - RT AVG IMP III; Lk 1

RS Legende/Bild: LIBERO PATRI; Bacchus links stehend hält Oinochoe über Panther; Thyrsusstab

Münzstätte: Rom

Besonderheiten: -

Münzzeichen:

Referenz: RIC 32

Prägeperiode: 194 n. Chr.

Stempelstellung: $1,79 \mathrm{~g} 12^{\mathrm{h}}$

\section{Kat. 48}

ohne Abb.

Nominal: D

AO: Lungauer Heimatmuseum Tamsweg, o. Inv.

Prägeherr: Antoninus III (Caracalla) für Iulia Domna: IVLIA

PIA FELIX AVG; Pal

RS Legende/Bild: VENVS GENETRIX; Venus sitzt links, hält Apfel und Szepter, zu ihren Füßen Cupido

Münzstätte: Rom

Besonderheiten: -

Münzzeichen:

Referenz: RIC $389 b$

Prägeperiode: 211-217 n. Chr.

Stempelstellung: $1,5 \mathrm{~g} 1^{\mathrm{h}}$
Kat. 49

ohne Abb.

Nominal: Ant

AO: Lungauer Heimatmuseum Tamsweg, o. Inv.

Prägeherr: Gordianus III (238-244 n. Chr.) IMP GORDIANVS PIVS FEL AVG; Stk2PCv

RS Legende/Bild: ORIE - N - S AVG; Sol, stehend links, in der Rechten Globus

Münzstätte: Antiochia

Besonderheiten: -

Münzzeichen:

Referenz: RIC 213

Prägeperiode: $242-244 \mathrm{n}$. Chr.

Stempelstellung: $2,79 \mathrm{~g} 6^{\mathrm{h}}$

Kat. 50

ohne Abb.

Nominal: As

AO: Lungauer Heimatmuseum Tamsweg, o. Inv.

Prägeherr: Prägeimitation Büste mit Bart nach rechts

RS Legende/Bild: Kanne

Münzstätte: Virunum (?) (Vorbild Rom)

Besonderheiten: -

Münzzeichen:

Referenz: RIC 32

Prägeperiode: ca. 138-235 n. Chr. (vor Severus Alexander [222-235 n. Chr.])

Stempelstellung: $13,11 \mathrm{~g} 12^{\mathrm{h}}$ 


\section{Abgekürzt zitierte Literatur}

Baatz - Herrmann 1982

Barkóczi 1988

Bishop - Coulston 2006

Deimel 1987

Fleischer 1998

Flügel 1993

Follman-Schultz 1992

Franken 1993

Franken 1995

Fremersdorf 1959

Garbsch 1992

Garbsch 1994

Geschwind 1998

Grönke - Weinlich 1992

Höck 2006

James 2004

Jobst 1974

Junkelmann 1992

Künzl 2003

Mackensen 1973

Mackensen 2001

Moucka-Weitzel 1998

Oldenstein 1976

Ortisi 2002

Radman-Livaja 2004

Riha 1979

Ritterling 1912

Rütti 1988

Schleiermacher 2000

Schütz 2003

Sedlmayer 1995

Sipmson 2000

Stadler 1995
D. Baatz - F. R. Herrmann, Die Römer in Hessen (Stuttgart 1982).

L. Barkóczi, Pannonische Glasfunde in Ungarn (Budapest 1988)

C. Bishop - J. C. N. Coulston, Roman Military Equipment. From the Punic Wars to the Fall of Rome ${ }^{2}$ (Oxford 2006).

M. Deimel, Die Bronzekleinfunde vom Magdalensberg, AForschMB 9 (Klagenfurt 1987).

R. Fleischer, Die Ergebnisse der Grabungen 1964-1970, in: R. Fleischer - V. Moucka-Weitzel, Die römische Straßenstation Immurium - Moosham im Salzburger Lungau, Archäologie in Salzburg 4 (Salzburg 1998) 9-51.

Ch. Flügel, Fibeln und Bronzegefäße von Kempten-Cambodunum, Cambodunumforschungen 5, Materialhefte zur bayerischen Vorgeschichte 63 (Kallmünz 1993).

A. B. Follmann-Schultz, Die römischen Gläser im Rheinischen Landesmuseum Bonn (Köln 1992).

N. Franken, Zur Typologie antiker Schnellwaagen, BJB 193, 1993, 69-120.

N. Franken, Die römischen Schnellwaagen im Rheinischen Landesmuseum Bonn, BJB 195, 1995, 425-438.

F. Fremersdorf, Römische Gläser mit Fadenauflage in Köln, Denkmäler des römischen Köln 5 (Köln 1959).

J. Garbsch, Römische Schnellwaagen mit hölzernem Balken, BayVgB1 57, 1992, 231-259.

J. Garbsch, Römische Schnellwaagen aus Bronze und Holz im Rheinischen Landesmuseum Trier, TrZ 57, 1994, 275-282.

M. Gschwind, Pferdegeschirrbeschläge der zweiten Hälfte des 3. Jahrhunderts aus Abusina/Eining, SaalburgJb 49, 1998, 112-138.

E. Grönke - E. Weinlich, Römische Laufgewichtswaagen, BayVgB1 57, 1992, 189-230.

A. Höck, Neues Militärisches aus Mechel, VLMTir 86, 2006, 245-276.

S. James, The Arms and Armour and other Military Equipment. Excavations at Dura Europos 1928-1937 (London 2004).

W. Jobst, Die römischen Fibeln aus Lauriacum, FiL 10 (Linz 1975).

M. Junkelmann, Die Reiter Roms III. Zubehör, Reitweise, Bewaffnung (Mainz 1992).

E. Künzl, Griechische Türen der klassischen und hellenistischen Zeit, in: E. und S. Künzl (Hrsg.), Das römische Prunkportal von Ladenburg, FBerBadWürt 94, 2003, 223-248.

M. Mackensen, Ein Fibelgrab von Regensburg-Großprüfening. Zu den Scheibenfibeln mit bronzenem Pressblechmedaillons, BayVgB1, 38, 1973, 57-79.

M. Mackensen, Militärische oder zivile Verwendung frühkaiserzeitlicher Pferdegeschirranhänger aus der Provinz Africa Proconsularis und den Nordwestprovinzen, Germania 79, 2001, 325-346.

V. Moucka-Weitzel, Die Kleinfunde von Immurium-Moosham, in: R. Fleischer - V. Moucka-Weitzel, Die römische Straßenstation Immurium - Moosham im Salzburger Lungau, Archäologie in Salzburg 4 (Salzburg 1998) 52-286.

J. Oldenstein, Zur Ausrüstung römischer Auxiliareinheiten. Studien zu Beschlägen und Zierrat an der Ausrüstung der römischen Auxiliareinheiten des obergermanisch-raetischen Limesgebietes aus dem zweiten und dritten Jahrhundert n. Chr., BerRGK, 57, 1976, 49-284.

S. Ortisi, Die früh- und mittelkaiserzeitlichen Fibeln, in: S. Ortisi - Ph. Pröttel, Römische Kleinfunde aus Burghöfe, Frühgeschichtliche und provinzialrömische Archäologie. Materialien und Forschungen 6 (Rahden/Westfalen 2002) 9-84.

I. Radman-Livaja, Militaria Siciensia, Nalazi rimske vojne opreme iz Siska u fundusu Arheološkoga muzeja u Zagrebu, Musei Archeologici Zagrebiensis Catalogi et Monographiae 1 (Zagreb 2004).

E. Riha, Die römischen Fibeln aus Augst und Kaiseraugst, FiA 3 (Augst 1979).

E. Ritterling, Das frührömische Lager bei Hofheim im Taunus, Annalen des Vereins für Nassauische Altertumskunde 40 (Wiesbaden 1912).

C. B. Rütti, Die Gläser, Beiträge zum römischen Oberwinterthur - Vitudurum 4, Berichte der Zürcher Denkmalpflege, Monographien 5 (Zürich 1988).

M. Schleiermacher, Römisches Pferdegeschirr aus den Kastellen Saalburg, Zugmantel und Feldberg, SaalburgJb 50, 2000, 167-193.

N. M. Schütz, Eisenfunde aus der Stadt auf dem Magdalensberg II, AForschMB 14 (Klagenfurt 2003).

H. Sedlmayer, Die römischen Fibeln von Wels. Quellen und Darstellungen zur Geschichte von Wels, Sonderreihe des Musealvereines Wels 4 (Wels 1995).

G. Simpson, Roman Weapons, Tools, Bronze Equipment and Brooches from Neuss - Novesium. Excavations 1955-1972, BAR IntSer 862 (Oxford 2000).

H. Stadler mit einem Beitrag von Oeggl, Die Ausgrabungen auf der Burgruine Flaschberg bei Oberdrauburg in Kärnten, in: K. Karpf u. a., Flaschberg. Archäologie und Geschichte einer mittelalterli- 
Unz - Deschler-Erb 1997

Vanden Berghe 1996 chen Burganlage bei Oberdrauburg in Kärnten, Nearchos 3, 1995, 137-333.

Ch. Unz - E. Deschler-Erb, Katalog der Militaria aus Vindonissa, Pro Vindonissa 14 (Brugg 1997).

L. Vanden Berghe, Some Roman military equipment of the first three centuries AD in Belgian museums, JRomMilSt 7, 1996, 59-93.

Dr. Raimund Kastler, MAS

Salzburg Museum, Postfach 10, Mozartplatz 1, A-5010 Salzburg

E-Mail: raimund.kastler@salzburgmuseum.at

\section{Dr. Matthias Pfisterer}

Kunsthistorisches Museum, Burgring 5, A-1010 Wien

E-Mail: matthias.pfisterer@khm.at

Abbildungsnachweis: Abb. 1: Photo M. Pfisterer; Abb. 2-13: Zeichnung F. Krois, Salzburg Museum. 